\title{
APPLICATION OF THE REY-OSTERRIETH COMPLEX FIGURE TEST FOR ASSESSMENT OF COGNITIVE IMPAIRMENT IN MULTIPLE SCLEROSIS
}

\author{
Ivan Dimitrov ${ }^{1}, V_{y a r a} K_{i r k o v a}{ }^{2}$, Ara Kaprelyan ${ }^{3}$, Borislav Ivanov ${ }^{4}$, Nataliya Usheva ${ }^{5}$, \\ Margarita Grudkova ${ }^{3}$, Daniela Arabadzhieva ${ }^{3},{ }^{3}$ Nadezhda Deleva ${ }^{3}$ \\ ${ }^{1}$ Head of Affiliate Sliven, Medical University of Varna \\ ${ }^{2}$ Stroke Unit, Second Neurology Clinic, St. Marina University Hospital, Varna \\ ${ }^{3}$ Department of Neurological Diseases and Neuroscience, ${ }^{4}$ Department of Clinical Medical \\ Sciences, Dental Faculty, ${ }^{5}$ Department of Social Medicine and Health Care Organisation, \\ Medical University of Varna
}

\begin{abstract}
INTRODUCTION: Cognitive impairment is frequent in multiple sclerosis and predominantly affects visuospatial abilities, memory, attention, and executive functions. Because of the specific cognitive profile, different from that in Alzheimer's disease and other frequent disorders presenting with dementia, specific neuropsychological instruments need to be used in multiple sclerosis. The aim of the present study is to assess the applicability of the Rey-Osterrieth complex figure test (ROCFT) for assessing cognitive impairment in patients with multiple sclerosis in comparison with a control group.

MATERIAL AND METHODS: One hundred and two individuals, 70 patients with MS and 32 healthy control subjects were assessed using ROCFT. A standardized system for assessment by points and percentages was used. The results from both groups were compared statistically using independent samples t-test.

RESULTS: On the copying task, the differences between patients and controls reached statistical significance, $p<0.05$ for the raw score, and $\mathbf{p}<\mathbf{0 . 0 0 1}$ after results were grouped by percentiles. On the drawing by memory task the differences between the two groups also showed statistical significance, $p<0.01$.

CONCLUSION: ROCFT shows statistically significant differences between patients with multiple sclerosis and healthy subjects. Highest levels of significance were observed for copying, scored by percentiles, and for drawing by memory. The application of ROCFT can provide valuable information about cognitive dysfunction in multiple sclerosis.
\end{abstract}

Keywords: cognitive impairment, multiple sclerosis, ROCFT, visuospatial abilities

Address for correspondence:

Assoc. Prof. Ivan Dimitrov, $M D, P h D$

St. Marina University Hospital of Varna

Medical University of Varna

1, Hristo Smirnenski Str.

9010 Varna, Bulgaria

e-mail:indimitrov@yahoo.com

Received: August 13, 2015

Accepted: September 15, 2015

\section{INTRODUCTION}

Cognitive impairment is typical of multiple sclerosis (MS) and can be found in 40 to $65 \%$ of the patients (1). According to some researchers, even up to $70 \%$ of the MS population is affected by cognitive impairment (2). The cognitive profile of MS patients is dominated by disturbances of information processing speed, episodic memory, and some aspects of executive dysfunction (3). Literature reports pro- 
Application of the rey-osterrieth complex figure test for assessment of cognitive impairment in multiple sclerosis

vide evidence of heterogeneity in the cognitive performance among patients, which reflects the influence of the clinical course of the disease, but also of numerous factors such as genetics, sex, intelligence, neuropsychiatric comorbidity, etc (3). Because of the characteristic cognitive profile, showing differences from that in Alzheimer's disease and other frequent disorders presenting with dementia, specific neuropsychological instruments are subject to research and application in MS (1).

The Rey-Osterrieth complex figure test (ROCFT) was introduced by the Swiss psychologist André Rey in 1941, and standardized by Paul-Alexandre Osterrieth in $1944(4,5)$. To do the test, subjects are required to copy a complex figure with 18 elements first, and then to draw the same figure by memory. Different cognitive functions can thus be examined, including visuospatial abilities, memory, attention, and executive functions $(6,7)$. Visuospatial construction is a complex cognitive process which includes several components: purely constructional, visuoperceptual, attention-related and decision making (8). Executive functions are known to influence the encoding and retrieval of visuospatial information (9). Graphical confabulations have been described during the drawing from memory component of ROCFT, the complex figure being drawn as a church, a fish, a human figure, etc. by patients with dementia (10). Such confabulations are thought to result mainly from the deficit of visual memory.

ROCFT is classically used in dementia and mild cognitive impairment (11). It has been reported that the addition of a nonverbal memory and executive functioning test, such as ROCFT, to the standard mini-mental state examination could improve the detection of mild cognitive impairment (12). Moreover, in a study of nondemented older adults, ROCFT reproduction score was found to correlate moderately with hippocampal volumes (13). The test has also been used for assessment of specific cognitive disturbances in other disorders. Garcia-Casares et al. assessed patients with type 2 diabetes mellitus and found that they performed weaker than control subjects (14). Park et al. included the test in their protocol for assessment of children with attention deficit hyperactivity disorder (ADHD) (15), while Martens et al. used it for studying the neurocognitive development of children (16). According to Schreiber et al. the test can detect persisting executive dysfunction in adults with ADHD (17). Using ROCFT, Heled et al. found executive dysfunction in patients with anorexia nervosa, even after restoration of weight (18). In a recent study of MS patients, using ROCFT as one of the neuropsychological instruments in a hippocampal dysfunction-oriented battery, together with volumetric assessment, Longoni et al. demonstrated that damage to the CA1 and subiculum was significantly correlated to test performance (19). Ge et al. used ROCFT in a neuropsychological test battery in patients with MS and found a moderate but significant correlation between the magnetic field correlation value in the thalamus and the test results (20). Gmeindl and Courtney also included ROCFT in their study of spatial working memory in MS (21). The test was administered to MS patients and control participants and revealed significant differences between groups, but only in the time required for its completion. Liguori et al. investigated visuospatial memory in MS patients using a battery of tests including ROCFT with immediate and delayed recall (22). ROCFT was among the instruments which showed significant differences between the cognitive performances of patients and controls. Moreover, grey matter volumes of MS patients were positively correlated to ROCFT scores.

According to Yamashita et al., handedness may influence test performance which can be lower if the non-dominant hand is used in the copy trial, regardless of the hand used during the drawing by memory task (23). The authors underline the clinical importance of this finding, particularly in patients who draw using their non-dominant hand because of motor deficit of the dominant one, which can be the case in some patients with MS.

Different scoring systems have been developed for the ROCFT (24-27). Among the most commonly used are the classical Osterrieth 36-point system and the Boston Qualitative Scoring System (BQSS). The latter includes five different scores: Planning, Fragmentation, Neatness, Perseveration, and Organization (28). In a study of Elderkin-Thompson et al. the BQSS summary drawing scores correlated well with the Osterrieth summary scores (24). ROCFT score may be influenced by age, gender, and intelligence (29). The order in which the figure is drawn can also 
Ivan Dimitrov, Vyara Kirkova, Ara Kaprelyan et al.

be monitored. Usually this is done using pen switching or the flowchart method (30).

Alternatives to ROCF have been developed, such as the Modified Taylor Complex Figure (MTCF), which is considered similar to ROCF in terms of copy and recall scores (31), unlike the original Taylor Complex Figure, which may be easier to recall than the ROCFT (32).

The aim of the present study is to evaluate the applicability of ROCFT for assessing cognitive disturbance in patients with MS, by comparison with the results of a control group.

\section{MATERIAL AND METHODS}

One hundred and two individuals, 70 patients with MS and 32 healthy control subjects were assessed using ROCFT. They were recruited among the inpatients of First neurological clinic, St. Marina University Hospital, Varna, Bulgaria. Informed consent form was signed by all participants. The test was completed by initial copying of the complex figure, followed by drawing by memory after a three-minute interval. The traditional method for scoring by points and percentiles was used for both trials. First, each of the 18 elements of the complex figure was scored individually with a maximum of 2 points if drawn and placed correctly, 1 point if drawn correctly but placed improperly or drawn incorrectly but recognizable and placed properly, 0.5 points if drawn incorrectly but recognizable and placed poorly, and 0 points if absent or not recognizable. Results for the single elements were then summed up to obtain a score for the whole figure, up to 36 points. Scores

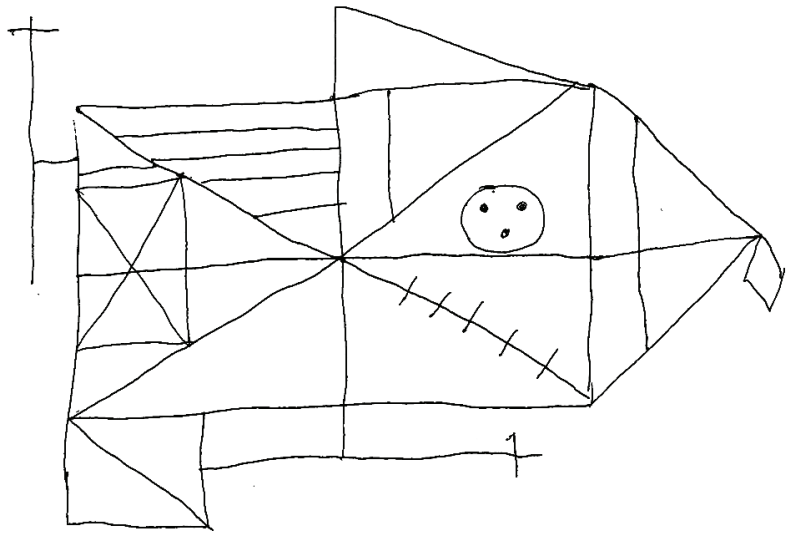

were converted into percentiles according to conversion tables for the copy and memory trials. The time for completion and the order in which the figure was drawn by the subjects were not taken into account in this study.

The results of both groups of participants, MS patients and healthy subjects, were compared statistically using independent samples t-test.

\section{RESULTS}

The test was completed by all participants. On the copying task patients obtained $33.3 \pm 6.3$ points, while the control group scored $35.4 \pm 1.3$ points, the maximum score being 36 . The differences between the two groups reached statistical significance, $\mathrm{p}<0.05$. After the results were grouped by percentiles, statistical significance was higher, $\mathrm{p}<0.001$. On the drawing by memory task patients scored $19.7 \pm 7.2$ points, and control subjects scored $24.5 \pm 6.7$ points.

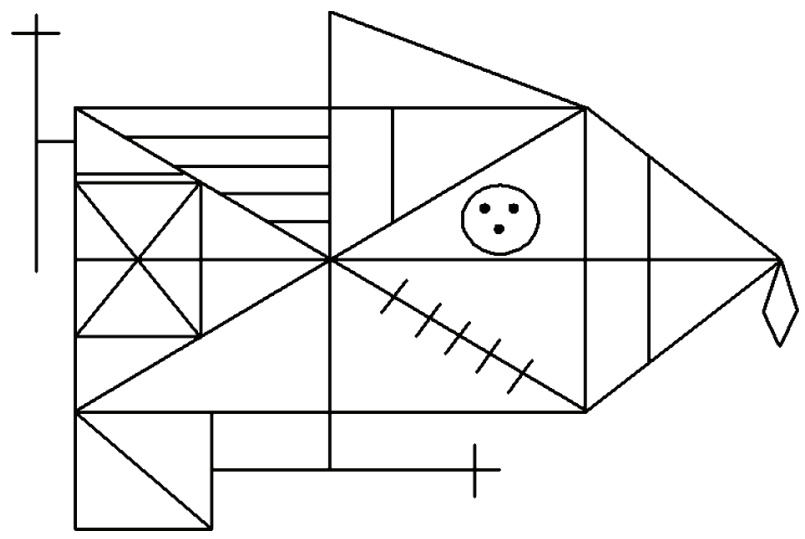

Fig. 1. Rey-Osterrieth complex figure, original

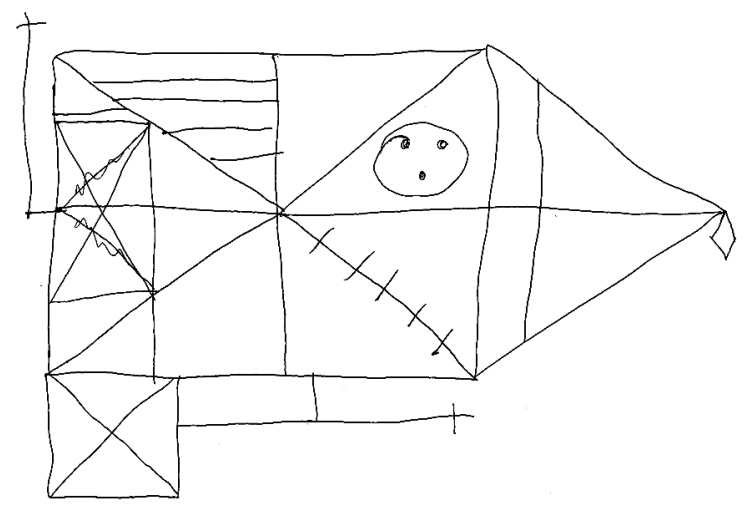

Fig. 2. Copying (left) and drawing by memory (right) of the ROCF by a control subject, male, 37 yrs. 

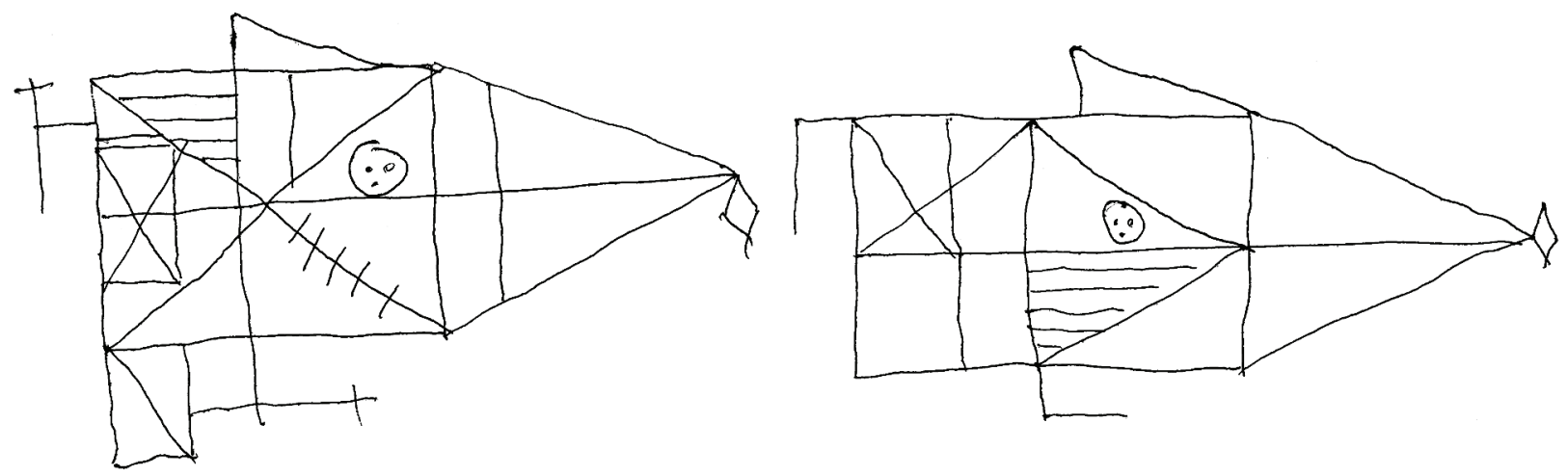

Fig. 3. Copying (left) and drawing by memory (right) of the ROCF by a MS patient, male, 43 yrs., EDSS 2.5
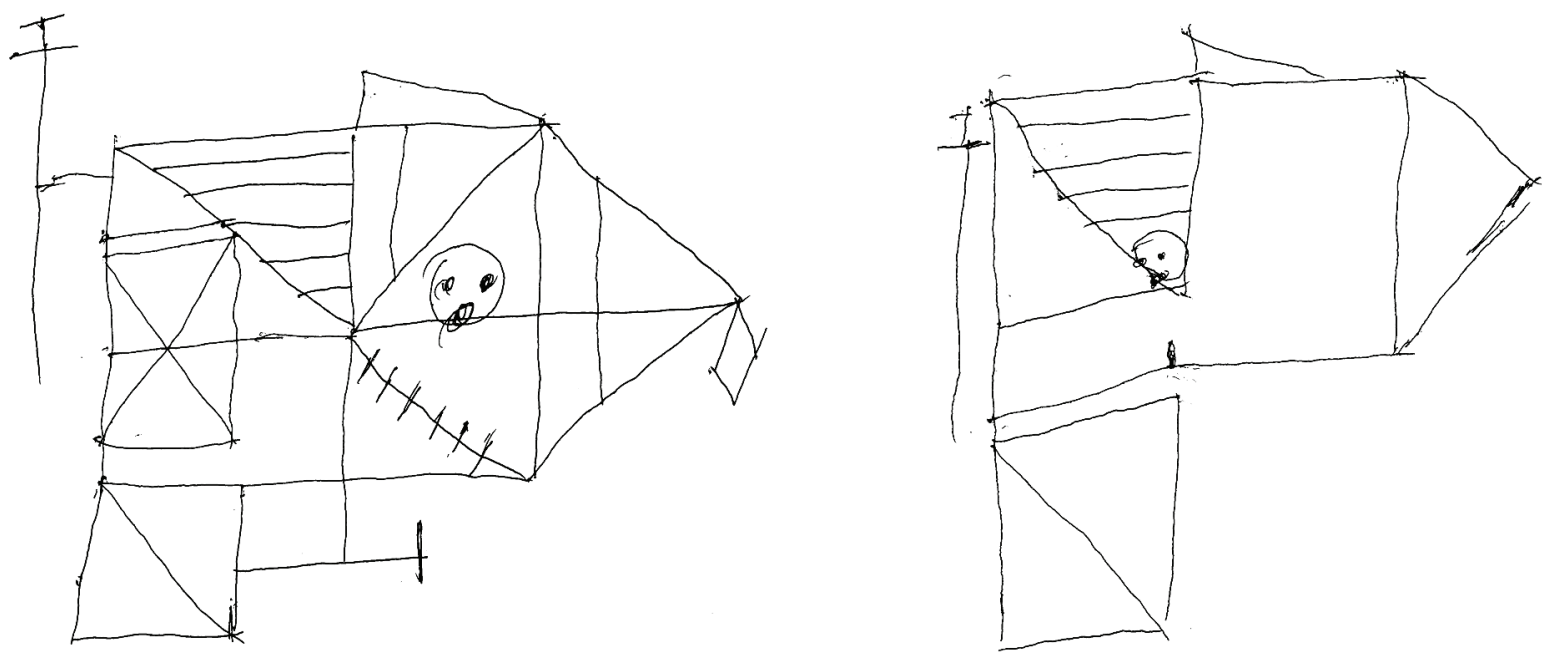

Fig. 4. Copying (left) and drawing by memory (right) of the ROCF by a MS patient, female, 36 yrs., EDSS 4.5

The differences between the two groups were statistically significant, $\mathrm{p}<0.01$. Patients and controls did not differ significantly regarding age and level of education $(\mathrm{P}>0.05)$.

The original Rey-Osterrieth figure, as well as examples of copying and drawing by memory by representatives of the patients and the control group are shown on Fig.1-4.

\section{DISCUSSION}

In this study we have applied a well-known neuropsychological instrument in patients with MS and control subjects. Even though its properties of assessing visuospatial abilities, memory, attention, and executive functions, correspond to the cognitive functions which are considered impaired in multiple sclerosis, its use in this domain is not widespread.

In order to simplify the test administration and scoring, we did not take into account the order in which the figure was drawn, and used the traditional scoring method, instead of more complicated scoring systems. We found it to be easy to use, yet informative. In the population we assessed, ROCFT demonstrated statistically significant differences between patients with MS and healthy subjects, the most pronounced being figure copying, scored according to the method of the percentiles, and drawing by memory. We therefore encourage not only the use of raw scores, but also their conversion into percentiles, as this could complement the results. Such findings motivate an extensive use of the instrument in studies of cognitive impairment in MS. The application of ROCFT would provide valuable information about the specific cognitive impairment that develops in the course of the disease. 
Ivan Dimitrov, Vyara Kirkova, Ara Kaprelyan et al.

\section{CONCLUSION}

ROCFT is widely available, easy to apply, relatively easy to score, standardized and language independent. It is capable of revealing cognitive dysfunction in domains which are known to be affected in patients with multiple sclerosis. All these qualities allow us to encourage the use of the test in this patient population, and to support its inclusion in cognitive test batteries developed for multiple sclerosis.

\section{REFERENCES}

1. Strober LB, Rao SM, Lee JC, Fischer E, Rudick R. Cognitive impairment in multiple sclerosis: An 18 year follow-up study. Mult Scler Relat Disord. 2014 Jul;3(4):473-481.

2. Morgen K, Sammer G, Courtney SM, Wolters T, Melchior H, Blecker CR, et al. Evidence for a direct association between cortical atrophy and cognitive impairment in relapsing-remitting MS. Neuroimage. 2006 Apr 15;30(3):891-898.

3. Benedict RH, Zivadinov R. Risk factors for and management of cognitive dysfunction in multiple sclerosis. Nat Rev Neurol. 2011 Jun;7(6):332-342.

4. Rey A. L'examen psychologique dans les cas d'encephalopathie traumatique. Archives de Psychologie. 1941;28:215-285.

5. Osterrieth PA. Le test de copie d'une figure complex: Contribution a l'etude de la perception et de la memoire. Archives de Psychologie. 1944;30:286-356.

6. Caffarra P, Vezzadini G, Dieci F, Zonato F, Venneri A. Rey-Osterrieth complex figure: normative values in an Italian population sample. Neurol Sci. 2002 Mar;22(6):443-447.

7. Hubley AM, Tremblay D. Comparability of total score performance on the Rey-Osterrieth Complex Figure and a modified Taylor Complex Figure. J Clin Exp Neuropsychol. 2002 May;24(3):370-382.

8. Biesbroek JM, van Zandvoort MJ, Kuijf HJ, Weaver NA, Kappelle LJ, Vos PC, et al. The anatomy of visuospatial construction revealed by lesion-symptom mapping. Neuropsychologia. 2014 Sep;62:68-76.

9. Walker K, Schwehm A, Damewood G, Wellington R. A-53Understanding the Differential Contribution of Executive Functioning Processes to Visuospatial Memory. Arch Clin Neuropsychol. 2014 Sep;29(6):522-523.
10. Pelati O, Castiglioni S, Isella V, Zuffi M, de Rino F, Mossali I, et al. When Rey-Osterrieth's Complex Figure Becomes a Church: Prevalence and Correlates of Graphic Confabulations in Dementia. Dement Geriatr Cogn Dis Extra. 2011 Jan;1(1):372-380.

11. Isella V, Traficante D, Tagliabue CF, Mapelli C, Ferri F, Caffarra P, et al. A retrospective survey on rotated drawing in persons with mild cognitive impairment or degenerative dementia. Clin Neuropsychol. 2013;27(8):1300-1315.

12. Miller J, Hanson E, Baerresen K, Miller K, Gottuso A, Ercoli L, et al. A-12Screening for Mild Cognitive Impairment (MCI) with the Mini-Mental Status Exam (MMSE) and Rey-Osterrieth Complex Figure Test (ROCF). Arch Clin Neuropsychol. 2014 Sep;29(6):508.

13. Vyhnalek M, Nikolai T, Andel R, Nedelska Z, Rubinova E, Markova H, et al. Neuropsychological correlates of hippocampal atrophy in memory testing in nondemented older adults. J Alzheimers Dis. 2014;42 Suppl 3:S81-90.

14. Garcia-Casares N, Jorge RE, Garcia-Arnes JA, Acion L, Berthier ML, Gonzalez-Alegre P, et al. Cognitive dysfunctions in middle-aged type 2 diabetic patients and neuroimaging correlations: a cross-sectional study. J Alzheimers Dis. 2014;42(4):1337-1346.

15. Park S, Hong KE, Yang YH, Kang J, Park EJ, Ha K, et al. Neuropsychological and behavioral profiles in attention-deficit hyperactivity disorder children of parents with a history of mood disorders: a pilot study. Psychiatry Investig. 2014 Jan;11(1):65-75.

16. Martens R, Hurks PP, Jolles J. Organizational strategy use in children aged 5-7: standardization and validity of the Rey Complex Figure Organizational Strategy Score (RCF-OSS). Clin Neuropsychol. 2014;28(6):954-973.

17. Schreiber HE, Javorsky DJ, Robinson JE, Stern RA. Rey-Osterrieth Complex Figure performance in adults with attention deficit hyperactivity disorder: a validation study of the Boston Qualitative Scoring System. Clin Neuropsychol. 1999 Nov;13(4):509-520.

18. Heled E, Hoofien D, Bachar E, Cooper-Kazaz R, Gur E, Ebstein RP. Employing executive functions of perceptual and memory abilities in underweight and weight-restored anorexia nervosa patients. Eat Weight Disord. 2014 Dec;19(4):479-487. 
Application of the rey-osterrieth complex figure test for assessment of cognitive impairment in multiple sclerosis

19. Longoni G, Rocca MA, Pagani E, Riccitelli GC, Colombo B, Rodegher M, et al. Deficits in memory and visuospatial learning correlate with regional hippocampal atrophy in MS. Brain Struct Funct. 2015 Jan;220(1):435-444.

20. Ge Y, Jensen JH, Lu H, Helpern JA, Miles L, Inglese $\mathrm{M}$, et al. Quantitative assessment of iron accumulation in the deep gray matter of multiple sclerosis by magnetic field correlation imaging. AJNR Am J Neuroradiol. 2007 Oct;28(9):1639-1644.

21. Gmeindl L, Courtney SM. Deconstructing spatial working memory and attention deficits in multiple sclerosis. Neuropsychology. 2012 Jan;26(1):57-70.

22. Liguori M, Fera F, Gioia MC, Valentino P, Manna I, Condino F, et al. Investigating the role of brainderived neurotrophic factor in relapsing-remitting multiple sclerosis. Genes Brain Behav. 2007 Mar;6(2):177-183.

23. Yamashita H. Right- and left-hand performance on the Rey-Osterrieth Complex Figure: A preliminary study in non-clinical sample of right handed people. Arch Clin Neuropsychol. 2010 Jun;25(4):314-317.

24. Elderkin-Thompson V, Boone KB, Kumar A, Mintz J. Validity of the Boston qualitative scoring system for the Rey-Osterrieth complex figure among depressed elderly patients. J Clin Exp Neuropsychol. 2004 Aug;26(5):598-607.

25. Frank J, Landeira-Fernandez J. Comparison between two scoring systems of the Rey-Osterrieth Complex Figure in left and right temporal lobe epileptic patients. Arch Clin Neuropsychol. 2008 Nov-Dec;23(7-8):839-845.

26. Ogino T, Watanabe K, Nakano K, Kado Y, Morooka T, Takeuchi A, et al. Predicting executive function task scores with the Rey-Osterrieth Complex Figure. Brain Dev. 2009 Jan;31(1):52-57.

27. Smith SR, Chang J, Schnoebelen KJ, Edwards JW, Servesko AM, Walker SJ. Psychometrics of a simple method for scoring organizational approach on the Rey-Osterrieth complex figure. J Neuropsychol. 2007 Mar;1(Pt 1):39-51.

28. Somerville J, Tremont G, Stern RA. The Boston Qualitative Scoring System as a measure of executive functioning in Rey-Osterrieth Complex Figure performance. J Clin Exp Neuropsychol. 2000 Oct;22(5):613-621.
29. Gallagher C, Burke T. Age, gender and IQ effects on the Rey-Osterrieth Complex Figure Test. Br J Clin Psychol. 2007 Mar;46(Pt 1):35-45.

30. Ruffolo JS, Javorsky DJ, Tremont G, Westervelt HJ, Stern RA. A comparison of administration procedures for the Rey-Osterrieth Complex Figure: flowcharts versus pen switching. Psychol Assess. 2001 Sep;13(3):299-305.

31. Hubley AM, Jassal S. Comparability of the ReyOsterrieth and Modified Taylor Complex Figures using total scores, completion times, and construct validation. J Clin Exp Neuropsychol. 2006 Nov;28(8):1482-1497.

32. Kuehn SM, Snow WG. Are the Rey and Taylor figures equivalent? Arch Clin Neuropsychol. 1992 Oct;7(5):445-448. 